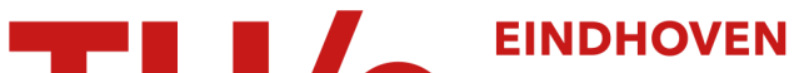 \\ UNIVERSITY OF \\ TECHNOLOGY
}

\section{Overlap equivalence in the Edwards-Anderson model}

Citation for published version (APA):

Contucci, P., Giardinà, C., Giberti, C., \& Vernia, C. (2006). Overlap equivalence in the Edwards-Anderson model. Physical Review Letters, 96(21), 217204-1/4. [217204]. https://doi.org/10.1103/PhysRevLett.96.217204

DOI:

10.1103/PhysRevLett.96.217204

Document status and date:

Published: 01/01/2006

\section{Document Version:}

Publisher's PDF, also known as Version of Record (includes final page, issue and volume numbers)

\section{Please check the document version of this publication:}

- A submitted manuscript is the version of the article upon submission and before peer-review. There can be important differences between the submitted version and the official published version of record. People interested in the research are advised to contact the author for the final version of the publication, or visit the $\mathrm{DOI}$ to the publisher's website.

- The final author version and the galley proof are versions of the publication after peer review.

- The final published version features the final layout of the paper including the volume, issue and page numbers.

Link to publication

\section{General rights}

Copyright and moral rights for the publications made accessible in the public portal are retained by the authors and/or other copyright owners and it is a condition of accessing publications that users recognise and abide by the legal requirements associated with these rights.

- Users may download and print one copy of any publication from the public portal for the purpose of private study or research.

- You may not further distribute the material or use it for any profit-making activity or commercial gain

- You may freely distribute the URL identifying the publication in the public portal.

If the publication is distributed under the terms of Article $25 \mathrm{fa}$ of the Dutch Copyright Act, indicated by the "Taverne" license above, please follow below link for the End User Agreement:

www.tue.nl/taverne

\section{Take down policy}

If you believe that this document breaches copyright please contact us at:

openaccess@tue.nl

providing details and we will investigate your claim. 


\title{
Overlap Equivalence in the Edwards-Anderson Model
}

\author{
Pierluigi Contucci, ${ }^{1}$ Cristian Giardinà, ${ }^{2}$ Claudio Giberti, ${ }^{3}$ and Cecilia Vernia ${ }^{4}$ \\ ${ }^{1}$ Università di Bologna, Piazza di Porta S. Donato 5, 40127 Bologna, Italy \\ ${ }^{2}$ Eurandom, P.O. Box 513-5600, MB Eindhoven, The Netherlands \\ ${ }^{3}$ Università di Modena e Reggio Emilia, via Fogliani 1, 42100 Reggio Emilia, Italy \\ ${ }^{4}$ Università di Modena e Reggio Emilia, via Campi 213/B, 41100 Modena, Italy
}

(Received 27 October 2005; published 1 June 2006)

\begin{abstract}
We study the relative fluctuations of the link overlap and the square standard overlap in the threedimensional Gaussian Edwards-Anderson model with zero external field. We first analyze the correlation coefficient and find that the two quantities are uncorrelated above the critical temperature. Below the critical temperature we find that the link overlap has vanishing fluctuations for fixed values of the square standard overlap and large volumes. Our data show that the conditional variance scales to zero in the thermodynamic limit. This implies that, if one of the two random variables tends to a trivial one (i.e., deltalike distributed), then the other does also, and as a consequence, the "trivial-nontrivial" picture should be dismissed. Our results show that the two overlaps are completely equivalent in the description of the low temperature phase of the Edwards-Anderson model.
\end{abstract}

DOI: 10.1103/PhysRevLett.96.217204

PACS numbers: 75.10.Nr, 75.50.Lk

The low-temperature phase of short-range spin glasses is among the most unsettled problems in condensed matter physics [1,2]. To study its nature an order parameter was originally proposed by Edwards and Anderson [3], the disorder average of the local squared magnetization

$$
q_{\mathrm{EA}}=\operatorname{Av}\left(\omega_{i}^{2}\right)=\operatorname{Av}\left(\left[\frac{\sum_{\sigma} \sigma_{i} e^{-\beta H_{\sigma}}}{\sum_{\sigma} e^{-\beta H_{\sigma}}}\right]^{2}\right)
$$

which coincides with the quenched expectation of the local standard overlap of two spin configurations drawn according to two copies of the equilibrium state carrying identical disorder

$$
\operatorname{Av}\left(\omega_{i}^{2}\right)=\left\langle q_{i}\right\rangle=\operatorname{Av}\left(\frac{\sum_{\sigma, \tau} \sigma_{i} \tau_{i} e^{-\beta\left(H_{\sigma}+H_{\tau}\right)}}{\sum_{\sigma, \tau} e^{-\beta\left(H_{\sigma}+H_{\tau}\right)}}\right) .
$$

The previous parameter should reveal the presence of frozen spins in random directions at low temperatures. While that choice of the local observable is quite natural, it is far from unique; one can consider, for instance, the two point function $\operatorname{Av}\left(\omega_{i j}^{2}\right)$. In the case of the nearest-neighbor correlation function this yields the quenched average of the local link overlap.

When summed over the whole volume, the link overlap and the standard overlap give rise to a priori different global order parameters. In the mean-field case the two have a very simple relation: In the Sherrington-Kirkpatrick (SK) model, for instance, it turns out that the link overlap coincides with the square power of the standard overlap up to thermodynamically irrelevant terms. But in general, especially in the finite dimensional case of nearest-neighbor interaction like the Edwards-Anderson (EA) model, the two previous quantities have a different behavior with respect to spin flips: when summed over regions the first undergoes changes of volume sizes after spin flips, while the second is affected only by surface terms.

From the mathematical point of view, their role is also quite different. The square of the standard overlap represents, in fact, the covariance of the Hamiltonian function for the SK model, while the link overlap is the covariance for the EA model. Two different overlap definitions are naturally related to two different notions of distance among spin configurations. It is an interesting question to establish if two distances are equivalent for the equilibrium measure in the large volume limit and, if yes, to what extent (see [4] for a broad discussion on overlap equivalence and its relation with ultrametricity). They could in fact be simply equivalent in preserving neighborhoods (topological equivalence), or they could preserve order among distances (metric equivalence). The a priori different properties of the two overlaps have also been discussed in relation to the different pictures [droplet [5], mean field [1], and trivial nontrivial (TNT) [6,7]] that have been proposed to describe the nature of the low-temperature spin-glass state. In this respect, the distributions of the two overlaps are expected to be deltalike (trivial distribution, droplet theory), to have support on a finite interval (nontrivial distribution, mean-field theory), or to have different behavior depending on which overlap is considered (trivial link-overlap distribution, nontrivial standard-overlap distribution, TNT theory).

In this Letter we consider the EA model in $d=3$, with Gaussian couplings and zero external magnetic field in periodic boundary conditions. We study the relative fluctuations of the link overlap with respect to the square of standard overlap. We use the parallel tempering (PT) algorithm to investigate lattice sizes from $L=3$ to $L=12$. For every size, we simulate at least 2048 disorder realizations. For the larger sizes we used 37 temperature values in 
the range $0.5 \leq T \leq 2.3$. The choice of the lowest temperature is related to the possibility to thermalize the large sizes, but our results are perfectly compatible with those obtained by Marinari and Parisi [8] at $T=0$ (see the last paragraph before the conclusions). The thermalization in the PT procedure is tested by checking the symmetry of the probability distribution for the standard overlap $q$ under the transformation $q \rightarrow-q$. Moreover, for the Gaussian coupling case, another thermalization test is available: the internal energy can be calculated as both the temporal mean of the Hamiltonian and - by exploiting integration by parts - the expectation of a simple function of the link overlap [9]. We checked that with our thermalization steps both measurements converge to the same value. All the parameters used in the simulations are reported in Table I.

We recall the basic definitions. For a three-dimensional lattice $\Lambda$ of volume $N=L^{3}$, the square of the standard overlap among two spin configurations $\sigma, \tau \in\{+1,-1\}^{N}$ is

$$
q^{2}(\sigma, \tau)=\left(\frac{1}{N} \sum_{i} \sigma_{i} \tau_{i}\right)^{2}
$$

The link overlap is instead obtained from the nearestneighbor spins, namely, for $b=(i, j)$ with $i, j \in \Lambda$, $|i-j|=1$, and $\sigma_{b}=\sigma_{i} \sigma_{j}$,

$$
Q(\sigma, \tau)=\frac{1}{3 N} \sum_{b} \sigma_{b} \tau_{b}
$$

First, we investigate the behavior of the correlation coefficient between $q^{2}$ and $Q$,

$$
\rho=\frac{\left\langle\left(q^{2}-\left\langle q^{2}\right\rangle\right)(Q-\langle Q\rangle)\right\rangle}{\sqrt{\left\langle\left(q^{2}-\left\langle q^{2}\right\rangle\right)^{2}\right\rangle\left\langle(Q-\langle Q\rangle)^{2}\right\rangle}} .
$$

This quantity will tell us in which range of temperatures the two random variables are correlated. In that range we further investigate the nature of the mutual correlation by studying their joint distribution and, in particular, the conditional distribution $P\left(Q \mid q^{2}\right)$ of $Q$ at fixed values of $q^{2}$. We are interested in understanding if a functional relation among the two quantities exists, i.e., if the variance of the conditional distribution shrinks to zero at large volumes

TABLE I. Parameters of the simulations: system size, number of sweeps used for thermalization, number of sweeps for measurement of the observables, number of disorder realizations (Nreal), number of temperature values allowed in the PT procedure, temperature increment, and minimum and maximum temperature values.

\begin{tabular}{cccccccc}
\hline \hline$L$ & Thermalization & Equilibrium & Nreal & $n_{\beta}$ & $\delta T$ & $T_{\min }$ & $T_{\max }$ \\
\hline $3-6$ & 50000 & 50000 & 2048 & 19 & 0.1 & 0.5 & 2.3 \\
8 & 50000 & 50000 & 2680 & 19 & 0.1 & 0.5 & 2.3 \\
10 & 70000 & 70000 & 2048 & 37 & 0.05 & 0.5 & 2.3 \\
12 & 70000 & 70000 & 2048 & 37 & 0.05 & 0.5 & 2.3 \\
\hline \hline
\end{tabular}

and around what curve the conditional distribution is peaked. We have

$$
\begin{aligned}
P\left(Q \mid q^{2}\right) & =\frac{P\left(Q, q^{2}\right)}{P\left(q^{2}\right)} \\
& =\frac{\operatorname{Av}\left(\frac{\sum_{\sigma, \tau} \delta\left(Q-Q_{\sigma, \tau}\right) \delta\left(q^{2}-q_{\sigma, \tau}^{2}\right) e^{-\beta\left[H_{\sigma}+H_{\tau}\right]}}{\sum_{\sigma, \tau} e^{-\beta\left[H_{\sigma}+H_{\tau}\right]}}\right)}{\operatorname{Av}\left(\frac{\sum_{\sigma, \tau} \delta\left(q^{2}-q_{\sigma, \tau}^{2}\right) e^{-\beta\left[H_{\sigma}+H_{\tau}\right]}}{\sum_{\sigma, \tau} e^{-\beta\left[H_{\sigma}+H_{\tau}\right]}}\right)} .
\end{aligned}
$$

For this conditional distribution one could compute the generic $k$ th moment

$$
G_{k}\left(q^{2}\right):=\mathbb{E}\left(Q^{k} \mid q^{2}\right)=\int_{-1}^{1} Q^{k} P\left(Q \mid q^{2}\right) d Q .
$$

We are interested in the mean

$$
G\left(q^{2}\right):=G_{1}\left(q^{2}\right)=\mathbb{E}\left(Q \mid q^{2}\right)
$$

and the variance

$$
\operatorname{Var}\left(Q \mid q^{2}\right)=G_{2}\left(q^{2}\right)-G_{1}^{2}\left(q^{2}\right) .
$$

The method of the least squares immediately entails that the mean $G\left(q^{2}\right)$ is the best estimator for the functional dependence of $Q$ in terms of $q^{2}$. In fact, given any function $h\left(q^{2}\right)$, the mean of $\left[Q-h\left(q^{2}\right)\right]^{2}$ according to the joint distribution $P\left(Q, q^{2}\right)$ is $\sum_{i, j}\left[Q_{i}-h\left(q_{j}^{2}\right)\right]^{2} P\left(Q_{i}, q_{j}^{2}\right)=$ $\sum_{j} P\left(q_{j}^{2}\right) \sum_{i}\left[Q_{i}-h\left(q_{j}^{2}\right)\right]^{2} P\left(Q_{i} \mid q_{j}^{2}\right)$, where the sums run over all possible values of the random vector $\left(Q, q^{2}\right)$, which are finitely many on the finite system we simulated. Therefore, to minimize the mean, it suffices to minimize the inner sum, i.e., to choose $h\left(q^{2}\right)$ as the mean $G\left(q^{2}\right)$ of $Q$ with respect to the conditional distribution (6).

The scaling properties of the conditional variance (9) and the functional dependence (8) provide important information about the low-temperature phase of the model. Indeed, a vanishing variance in the thermodynamic limit implies that the two random variables $Q$ and $q^{2}$ do not fluctuate with respect to each other. If the functional dependence $G\left(q^{2}\right)$ among the two is a one-to-one increasing function, then it follows that the marginal probability distributions for the standard and link overlap must have similar properties. In particular, if one of the two is supported over a point, then the other must be so too.

We now describe our results. Figure 1 shows the correlation between the square standard overlap and the link overlap. The plot of Eq. (5) is done for different sizes of the system as a function of the temperature. It is clear from the figure that, as the system size increases, the correlation remains strong in the low-temperature region, while it becomes weaker in the high-temperature region. A sudden change in the infinite volume behavior of $\rho$ can be expected to occur close to the critical temperature $T_{c}$ of the model. The best estimate available in the literature-obtained through the analysis of the Binder parameter's curves of the variable $q^{2}$ for different system sizes - gives 


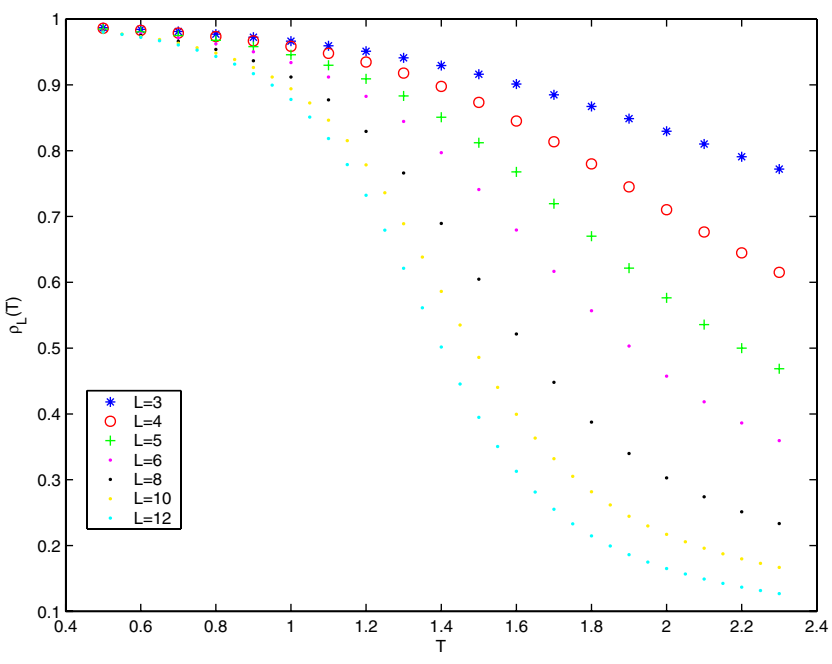

FIG. 1 (color online). $\quad \rho_{L}(T)$ as a function of the temperature $T$ for different sizes $L$ of the system.

$T_{c}=0.95 \pm 0.04$ [10] (we independently reproduced this estimate with our data for $q^{2}$ and obtained an estimate of $T_{c}=0.95 \pm 0.03$ ).

For each temperature, we did a fit of the data for $\rho$ to the infinite volume limit. We tried different scaling for the data, both exponential $\rho_{L}(T)=\rho_{\infty}(T)+a(T) e^{b(T) L}$ and power law $\rho_{L}(T)=\rho_{\infty}(T)+\alpha(T) L^{\beta(T)}$. The interesting information is contained in the asymptotic value $\rho_{\infty}(T)$. We measured the normalized $\chi^{2}$ for different values of $\rho_{\infty}(T)$ in the range $\left[0, \min _{L} \rho_{L}(T)\right]$, keeping $a(T)$ and $b(T)$ [or $\alpha(T)$ and $\beta(T)]$ as free parameters. In the region $T \geq$ 1.0 , we found that $\chi^{2}$ attains this minimal value for $\rho_{\infty}(T)=0$. For $T \leq 0.9$ the $\chi^{2}$ develops a sharp minimum corresponding to values $\rho_{\infty}(T) \neq 0$. The whole plot of the curve $\rho_{\infty}(T)$ as obtained from the best fit is represented in Fig. 2. Also, in the inset of the same figure the standard finite size scaling of the data is shown. We plot $\rho_{L}(T) L^{\psi / \nu}$

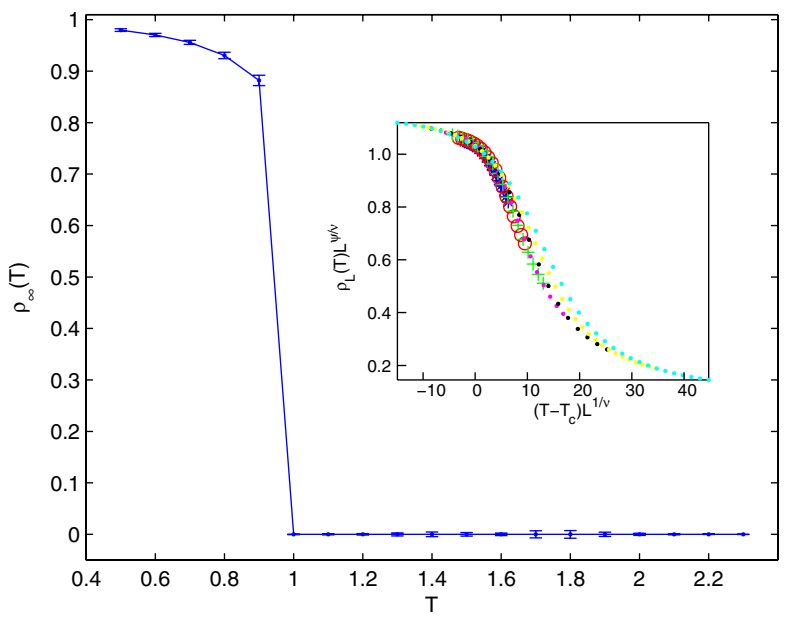

FIG. 2 (color online). $\rho_{\infty}(T)$ as a function of the temperature $T$. The finite size scaling is shown in the inset. versus the scaling variable $\left(T-T_{c}\right) L^{1 / \nu}$. A good scaling plot is obtained using $T_{c}=0.95, \nu=0.71$, and $\psi=$ 0.038 . The discrepancy between the value $\nu=2.0$ of Ref. [10] has to be attributed to the nonlinear relation between $Q$ and $q^{2}$ (see below). Figure 2 tells us that in the high-temperature phase the two random variables standard and link overlaps are asymptotically uncorrelated, while in the low-temperature one they display a nonvanishing correlation: within our available discrete set of temperature values, the temperature at which the correlation coefficient starts to be different from zero is in good agreement with the estimated critical value of the model.

We consider then the problem of studying the functional dependence (if any) between the two random variables $Q$ and $q^{2}$ in the low-temperature region. The points in Fig. 3 show the function $G\left(q^{2}\right)$ of Eq. (8) for different system sizes at $T=0.5$, well below the critical temperature. Also, we studied a third order approximation of the form $Q=$ $g\left(q^{2}\right)=a+b q^{2}+c q^{4}+d q^{6}$. Since we must have $Q=$ 1 for $q^{2}=1$, this actually implies $d=1-a-b-c$. The coefficients $a_{L, T}, b_{L, T}, c_{L, T}$ have been obtained by the least square method and then fitted to the infinite volume limit. The result is shown as continuous lines in Fig. 3. The good superposition of the curves to the data for $G\left(q^{2}\right)$ indicates that the functional dependence between the two overlaps is well approximated already at the third order.

Finally, we measured the variance Eq. (9) at low temperatures. We observed that the distribution is concentrating for large volumes around its mean value. The trend toward a vanishing variance for infinite system sizes is very clear. We analyzed all temperatures below $T_{c}$, and we found that the best fit of $\operatorname{Var}_{L}\left(Q \mid q^{2}\right)$, in terms of the $\chi^{2}$, is obtained by a power law of the form $a(T) L^{-b(T)}+c$, which gives $c=0$ for every value of the temperature. For

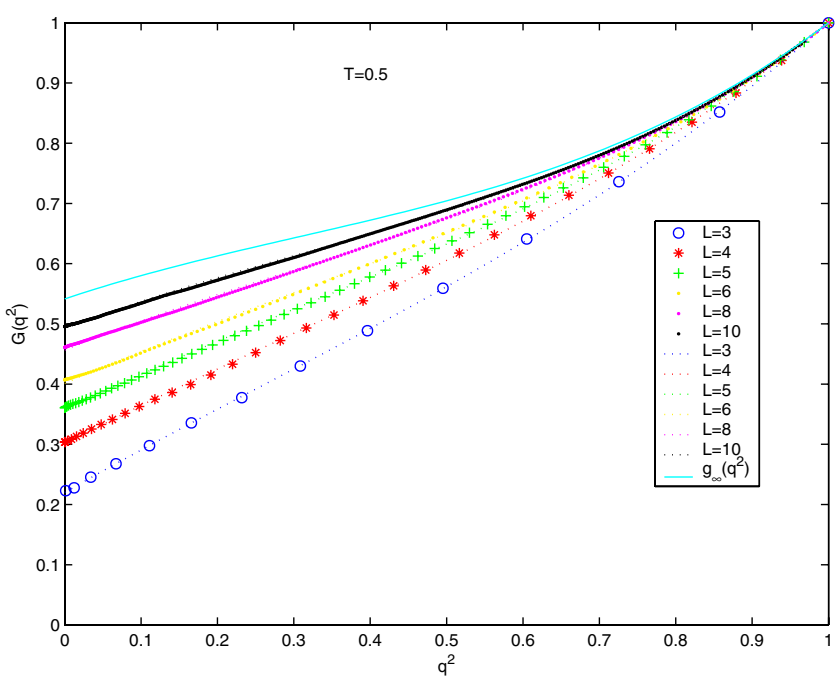

FIG. 3 (color online). Plot of the curves $g\left(q^{2}\right)$ (continuous lines) and of $G\left(q^{2}\right)$ (dotted lines) together with the infinite volume limit curve $g_{\infty}\left(q^{2}\right)$ (upper continuous line) for $T=0.5$. 


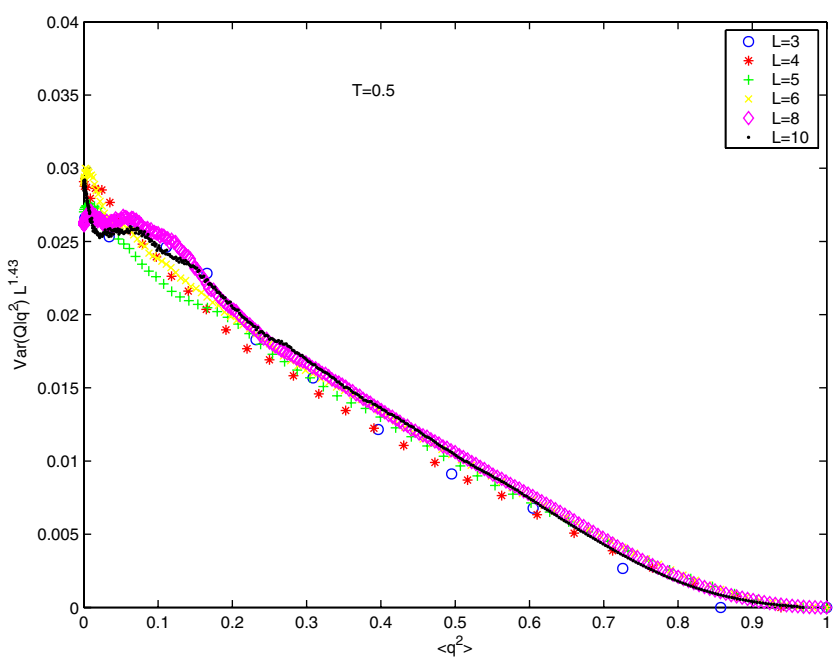

FIG. 4 (color online). $\quad L^{1.43} \operatorname{Var}_{L}\left(Q \mid q^{2}\right)$ as a function of $q^{2}$ for temperature $T=0.5$ and for different sizes $L$.

the lowest available temperature $T=0.5$, this is shown in Fig. 4 where we plot the data for $\operatorname{Var}_{L}\left(Q \mid q^{2}\right) L^{1.43}$ for different system sizes $L$ : all the different curves collapse to a single one. The data for other temperature values behave similarly, the only difference being that the coefficient $b(T)$ is increasing with the temperature (it stays in the range $[1.43,1.74]$ for $T \in[0.5,0.9])$. This result has quite strong consequences because it says that the two random variables $Q$ and $q^{2}$ cannot have different triviality properties: if one of them is trivial (deltalike distributed), the scaling law for their conditional variance implies that the other is also trivial. Our result then rules out the possibility to have a nontrivial standard overlap with a trivial link overlap as predicted, for instance, in the so-called TNT picture [6,7].

It is interesting to compare our result with previous works. Marinari and Parisi [8] have studied the relation $Q=[1-A(L)]+[A(L)-B(L)] q^{2}+B(L) q^{4}$ among the two overlaps at zero temperature, by ground state perturbation. We have extrapolated our data in the lowtemperature regime to zero temperature by a polynomial fit and then to the infinite volume limit $(L=\infty)$. The best fit for $L=\infty$ (i.e., the one with smaller $\chi^{2}$ ) is quadratic in $L^{-1}$. It gives $A=0.30 \pm 0.05\left(\chi^{2}=0.21\right)$, which is in agreement with the independent measure of Marinari and Parisi $\left(A=0.30 \pm 0.01, \chi^{2}=0.6\right)$. Note that their results are obtained with a complete different method than Monte Carlo simulations, namely, exact ground states.
Sourlas [11] studied the same problem in a different setting called the soft constraint model. Although a direct quantitative comparison is not possible with our method, his results are qualitatively similar. In the context of out-ofequilibrium dynamics, a strong correlation between the link overlap and the standard overlap in the lowtemperature phase was pointed out in Ref. [12].

In conclusion, our result shows quite clearly that, within the tested system sizes, the square of the standard overlap and the link overlap are totally equivalent as far as the quenched equilibrium state is concerned. In view of our result, the proposed pictures which assign different behavior to the two overlap distributions, in particular, the TNT $[6,7]$ picture, should be rejected. It is interesting to point out that, since the present analysis deals only with the distribution of $P\left(q^{2}, Q\right)$ and not with the higher order ones such as, for instance, $P\left(q_{1,2}^{2}, q_{2,3}^{2}, Q_{1,2}, Q_{2,3}\right)$, our results are compatible with different factorization properties of the two overlaps like those illustrated in [13].

We thank Enzo Marinari, Giorgio Parisi, and Federico Ricci-Tersenghi for interesting discussions. We also thank Cineca for the grant for computer time. C. Giardinà acknowledges NWO Project No. 613000435 for financial support.

[1] M. Mezard, G. Parisi, and M. A. Virasoro, Spin Glass Theory and Beyond (World Scientific, Singapore, 1987).

[2] C. M. Newman and D. L. Stein, cond-mat/0503345.

[3] S.F. Edwards and P.W. Anderson, J. Phys. F 5, 965 (1975).

[4] G. Parisi and F. Ricci-Tersenghi, J. Phys. A 33, 113 (2000).

[5] D. S. Fisher and D. A. Huse, Phys. Rev. Lett. 56, 1601 (1986).

[6] F. Krzakala and O.C. Martin, Phys. Rev. Lett. 85, 3013 (2000).

[7] M. Palassini and A.P. Young, Phys. Rev. Lett. 85, 3017 (2000).

[8] E. Marinari and G. Parisi, Phys. Rev. Lett. 86, 3887 (2001).

[9] P. Contucci, J. Phys. A 36, 10961 (2003).

[10] E. Marinari, G. Parisi, and J. J. Ruiz-Lorenzo, Phys. Rev. B 58, 14852 (1998).

[11] N. Sourlas, Phys. Rev. Lett. 94, 070601 (2005).

[12] S. Jimenez, V. Martin-Mayor, G. Parisi, and A. Tarancon, J. Phys. A 36, 10755 (2003).

[13] P. Contucci and C. Giardinà, Phys. Rev. B 72, 014456 (2005). 